\title{
Entre crematónimos y topónimos: los nombres de comercios
}

\author{
Carmen Fernández Juncal ${ }^{1}$ \\ Universidad de Salamanca, España
}

\begin{abstract}
Resumen
Los nombres de comercios constituyen una subcategoría dentro de los nombres propios y a su vez un tipo especial de crematónimos (Galkowski 2014) o ergónimos (Bauer 1985). Se trata de un tipo de vocablos que cuenta con escasos estudios, pero cuyo interés es indudable si consideramos la doble función que posee como una clase dentro de los nombres de marcas y como microtopónimos o topónimos urbanos.

El corpus del que partimos ha sido captado a partir de pruebas asociativas orales de disponibilidad léxica llevadas a cabo en un área perteneciente al llamado español central-peninsular, a partir de una muestra de hablantes seleccionados por cuota con afijación uniforme y considerando las variables género, edad y nivel sociocultural.

Una vez editados y procesados estadísticamente los datos, abordamos dos tareas: en primer lugar, caracterizamos el nuevo centro de interés, especialmente en contraste con otros crematónimos, los nombres de marcas, con los que comparten rasgos comunes, pero también atributos diferenciadores, precisamente por su carácter híbrido. En segundo lugar, analizamos cuantitativa y cualitativamente la distribución de los nombres de comercios en función de las variables
\end{abstract}

$1 \quad$ Para correspondencia, dirigirse a: Carmen Fernández Juncal (cjuncal@usal.es). Facultad de Filología. Plaza de Anaya, s/n. 37008 Salamanca, España. 
analizadas y describimos conceptual y lingüísticamente este tipo de vocablos desde el punto de vista de su alcance, las categorías productivas con las que están asociados, las lenguas empleadas y los recursos denominativos empleados en su creación.

Palabras clave: socionomástica, disponibilidad léxica, crematónimos, microtopónimos, ergónimos.

\section{BETWEEN CHREMATONYMS AND TOPONYMS: TRADE NAMES}

\section{Abstract}

Trade or business names are a subcategory of proper names and a special kind of chrematonyms (Galkowski 2017) or ergonyms (Bauer 1985). It is a type of vocabulary that has been little investigated until now, but whose interest is undoubted if we consider the dual function it has as a class within brand names and as microtoponyms or urban place names.

The corpus used for the present study has been compiled from oral associative tests of lexical availability carried out in an area belonging to the so-called central-peninsular Spanish, from a sample of speakers selected by quota with uniform affixation and considering the variables gender, age and sociocultural level.

Once the data have been edited and statistically processed, we tackle two tasks: firstly, we characterise the new centre of interest, especially in contrast to other chrematonyms, brand names, with which they share common features, but also differentiating attributes, precisely because of their hybrid character. Secondly, we analyse quantitatively and qualitatively the distribution of trade names according to the selected variables and we describe conceptually and linguistically this type of words from the point of view of their scope, the productive categories with which they are associated, the languages used and the denominational resources used in their creation.

Keywords: Socio-Onomastics, Lexical availability, chrematonyms, microtoponyms, ergonyms.

Recibido: 18/11/19

Aceptado: 08/06/20 


\section{INTRODUCCIÓN. LOS NOMBRES DE COMERCIOS}

Los nombres comerciales han presentado de siempre dificultades de análisis lingüístico. Como señalábamos en Fernández Juncal (en prensa), al contrario que los antropónimos y los topónimos, no han conseguido unificar en un tecnicismo la terminología que los agrupa: crematónimos (Galkowski 2014), ergónimos (Kryukova 2012 y, más específicamente para los rótulos de establecimientos, ecónimos o microtopónimos (Bajo Pérez 2002), urbanónimos, topónimos menores (Líbano Zumalacárregui 1993)², vocablos técnicos que presentan divergencias de significado y de enfoque. Deberíamos atribuir estas discrepancias a la diversidad interna de este conjunto de nombres propios, cuya categoría ha sido cuestionada (Fèvre-Pernet 2007), pero que no presenta dudas para la mayoría de los estudiosos en la materia (Schippan 1989, Vande Casteele 200123). Van Langendonck (2007) propone una escala de propiedad, desde los nombres propios prototípicos hasta los marginales pasando por lo no prototípicos, nivel donde se encuadrarían los que son nuestro objeto de estudio.

En la gramática académica los nombres de marcas se consideran nombres propios (como se deduce de Nueva gramática de la lengua española 12.8e), pero los nombres de empresas "se asimilan a los nombres propios" (12.8p) y no aparecen en la lista de tipos de topónimos (12.8ñ). En la Ortografia de la lengua española se separan claramente las marcas (4.2.4.11) de los establecimientos comerciales, cuya normativa sobre uso de mayúsculas se encuentra junto a los topónimos (4.2.4.7.5). Bajo Pérez (2002: 200) los considera nombres propios y los asimila a los topónimos mientras que Fernández Leborans (1999: 2.1.2) no los menciona abiertamente (quizás se podrían englobar entre "los nombres de productos de la actividad humana"), pero, en cualquier caso, los excluye de los nombres propios genuinos. Seide y Lucas (2018) los designan directamente como topónimos comerciales, aunando en esta denominación las dos vertientes de este tipo de signos. Finalmente, Kryukova (2012) les otorga un estatuto especial dentro de la

\footnotetext{
2 Esta proliferación de términos se complica si consideramos otras lenguas. Sírvanos de ejemplo la diversidad en inglés. Además de los señalados (ergonym, chrematonym, oekonym, microtoponym), tendríamos product name, brand name, commercial name, company name, firm name, trademark o business name.

Aunque, a tenor de la existencia de transformaciones constatadas de nombres propios en nombres comunes y viceversa, hay quien sostiene que estamos ante los extremos de un continuo dinámico (Gutknecht y Wehking 1985: 80).
} 
clase de los nombres, un cuerpo separado que se caracteriza por su adaptación a la lengua contemporánea y su fijación en la mente del receptor.

La disparidad de criterios en el análisis de los nombres de comercios se ha ido resolviendo de manera diferente en el ámbito legal ${ }^{4}$ : marcas, nombres comerciales, y nombres de comercios han sido tratados de manera conjunta en las legislaciones que los regulan, al menos en la española. Bien es cierto que en la Ley 32/1988, de 10 de noviembre, de Marcas, se distingue entre marcas, nombres comerciales y rótulos de establecimiento, que se definen como "el signo o denominación que sirve para dar a conocer al público un establecimiento y para distinguirlo de otros destinados a actividades idénticas o similares". Además de señalar los posibles elementos constitutivos de los mencionados rótulos, se señala que su registro, a pesar de ser de competencia municipal, está regido por las mismas normas que afectan a las marcas y nombres comerciales. Son estos dos conceptos los que constituyen el objeto de la nueva Ley 17/2001, de 7 de diciembre, de marcas, que, con modificaciones, es la que en la actualidad está vigente. En esta nueva norma, comprobado lo innecesario de la diferenciación ${ }^{5}$, los rótulos desaparecen como unidad propia y se asimilan a las marcas y nombres comerciales. Las primeras se definen como signos que sirven para distinguir productos o servicios en el mercado mientras que el nombre comercial sirve para identificar a una empresa en el tráfico mercantil y diferenciarla del resto de empresas del mismo o diferente sector. En el manual para posibles empresarios o creadores editado y actualizado en 2019 por el Ministerio de Industria, Comercio y Turismo y la Oficina Española de Patentes y Marcas se señala además otro concepto, el de denominación social, o nombre de persona jurídica, para cuya mejor explicación recurren al siguiente paralelo: "En un símil con la persona física la denominación social sería el "nombre civil" de la persona física y el nombre comercial sería el "nombre artístico" con que dicha persona se presenta a sus clientes en el mercado" (2019:4). Queda, por último, señalar al respecto que en ocasiones se emplea el mismo término para designar lo que son objetos con categoría jurídica y referencial diferente. Así, los productos con marca Massimo Dutti (denominación social Inditex S.A.) se venden en tiendas con ese mismo nombre.

La complejidad de los conceptos mencionados, derivada de la gran heterogeneidad en los casos detectados, no ha impedido que los crematónimos hayan sido estudiados desde diversos puntos de vista, fundamentalmente tres:

4 De hecho, Weyers (2007) propone partir de las especificaciones legales para poder hacer una clasificación onomástica general de este tipo de términos.

5 Comunicación personal de Fernando Carbajo Cascón, experto en Derecho Mercantil. 
en primer lugar, se trata de elementos con gran valor social y económico, ya que actúan como recursos dentro de la producción de bienes y servicios y desempeñan "a pivotal role in modern economies" (Boerrigter y Nijboer 2012: 3). El empleo de nombres de marca ha sido parte de la economía diaria desde antiguo, desde las señales en vasijas de barro o ganado hasta la explosión de los procedimientos mercadotécnicos a principios del XX, apoyados en un desarrollo industrial y de los transportes que hizo que se pudieran distribuir productos en áreas extensas en un periodo breve (Grandinetti 2008). En la actualidad no podemos imaginar una marca sin nombre, anónima (Smirnova 2017); no olvidemos que ponemos nombre a los objetos que nos importan, que poseen valor para nosotros (Sjöblom et al. 2013).

De esta perspectiva se deduce la segunda, de forma que el nombre comercial, como hemos visto, ha sido abordado desde el derecho, es decir, desde su protección y regulación, pero también desde la tutela del interés público en su uso (Teutsch 2012).

En tercer lugar, a pesar de la afirmación de Pottier (1954) de que "l'onomastique mineure doit avoir sa place", este tipo de nombres ha sido objeto del interés de los lingüistas en menor medida que otras áreas de la onomástica, de forma que constituye un capítulo arrinconado de los estudios léxicos (Moreu-Rey 1974), sobre todo, en el ámbito hispano. No es hasta finales del siglo XX cuando se observa el crecimiento de un interés por ellos (Kremer y Ronneberger-Sibold 2007), asociado a la importancia adquirida por estos nombres en otras esferas dado el indudable interés que poseen como ilustradores de los procesos sociales y económicos desarrollados en el último siglo (Leibring 2012).

Son dos las aproximaciones básicas que se han llevado a cabo desde nuestro ámbito: por un lado, tenemos los estudios de tipo estructural, que consideran los crematónimos como unidades léxicas, enfatizando los rasgos que los definen como nombres propios y su desempeño individualizador o distintivo. Según Fèvre-Pernet (2008), se trata de un enfoque formal ligado al contenido semántico, pero sin sistematizar la construcción de su sentido. Por otra parte, encontramos investigaciones de corte funcional y multidisciplinar, en las que estos términos se insertan dentro de un discurso que cumple diferentes cometidos (Kremer y Ronneberger-Sibold 2007, Sjöblom 2016), entre ellos la función comunicativa, expresiva y, sobre todo, la interacción emisor-cliente (Teutsch 2012): “o nome da loja exige uma rápida captação sensorial e, simultaneamente, visa uma forte interpelação ao público alvo e uma inscrição permanente na sua memória colectiva" (Moutinho y Coimbra 1998: 93). 
La onomástica comercial ${ }^{6}$ agrupa en su seno conjuntos de vocablos con diferentes niveles de fijación, que en el plano jurídico se caracterizan por estar generalmente registrados (Fèvre-Pernet y Roché 2005) y por integrarse en la competencia lingüística de los hablantes en términos semejantes a lo que ocurre con los nombres comunes (Fèvre-Pernet 2007). Desde el punto de vista de la socionomástica, los nombres de comercios poseen el atractivo de ser marcadores y espejos de procesos de carácter social cotidianos e históricos al mismo tiempo y realizaciones lingüísticas de normas y expectativas, de creencias y comportamientos (Li 2012). Además, nos proporcionan coordenadas para la descripción del entorno urbano en la misma línea en que se llevan a cabo los trabajos sobre paisaje lingüístico, del que son parte constituyente de primer orden. De hecho, en un proceso inverso, Puzey (2011) y Gorter (2014) señalan el paisaje lingüístico como una oportunidad para la investigación onomástica.

\section{CUESTIONES METODOLÓGICAS}

La presente investigación cuenta con los datos recabados a través de una encuesta de carácter asociativo llevada a cabo en la provincia de Salamanca en la primera mitad del año 2018. Salamanca se encuadra en el español centro-peninsular, variedad que se caracteriza, entre otros rasgos, por no estar afectada por la influencia de otras lenguas vecinas y por mantener un prestigio que la acompaña desde antiguo (Amorós Negre et al. 2012).

La muestra se ha diseñado por cuota de afijación uniforme, de manera que cada grupo resultante está compuesto por el mismo número de individuos, con un total de 60. La estratificación ha considerado las siguientes variables: en primer lugar, la edad, que sigue el modelo de segmentación del PRESEEA (http://preseea.linguas.net); en segundo lugar, el género, y, en tercer lugar, los estudios, que hemos categorizado entre universitarios y no universitarios. La distribución final de la muestra es la que detallamos en la tabla 1.

6 Pottier propuso apoteconimia, tecnicismo que no ha llegado a prosperar. 


\begin{tabular}{|cccccc|}
\hline & \multicolumn{2}{c}{ Hombres } & \multicolumn{2}{c|}{ Mujeres } & Total \\
& Univ & No Univ & Univ & No Univ & \\
\hline $\mathbf{2 0 - 3 4}$ & 5 & 5 & 5 & 5 & 20 \\
\hline $\mathbf{3 5 - 5 5}$ & 5 & 5 & 5 & 5 & 20 \\
\hline$>\mathbf{5 5}$ & 5 & 5 & 5 & 5 & 20 \\
\hline
\end{tabular}

Tabla 1. Distribución de la muestra

El cuestionario seguía las normas de los trabajos en disponibilidad léxica, pero comprendía varios centros de interés nunca tratados con anterioridad en torno al nombre propio: nombres de persona, nombres de ciudades, nombres de mujeres, nombres de países, nombres de hombre, nombres de marcas, nombres de comercios, nombres de familia (apellidos) y sobrenombres? Dado el perfil de los informantes, entre los que se incluían personas de edad avanzada y sin formación, con dificultades con la escritura, la prueba fue oral y, por lo tanto, individual. Esta modalidad de encuesta ofrece algunas dificultades, pero también algunas ventajas (Hernández Muñoz 2010). De acuerdo con experiencias y otros estudios previos (Frey Pereira 2007 y Manjón-Cabeza Cruz 2010), el tiempo de duración se fijó en un minuto. Hay que señalar que el contenido de los centros de interés no ha levantado dudas en los entrevistados, de forma que no fue necesario poner ejemplos. De hecho, y de manera extraordinaria respecto a los centros de interés empleados en este tipo de investigaciones, en nuestro caso no hay ningún rastro de vocablos periféricos o evocativos, que no se ajustasen al contenido de lo solicitado y ningún nombre común como tal.

El proceso de edición de los materiales presenta algunas disimilitudes respecto a las decisiones que se suelen llevar a efecto en el Proyecto Panhispánico de Léxico Disponible (www.dispolex.com). Estas discrepancias vienen dadas por los rasgos que caracterizan al nombre propio respecto al nombre común. En el caso del nombre propio su función es mayoritariamente referencial, de forma que ha sido considerado un designador rígido (Kripke 1981, Vande Casteele 2012). Este rasgo semántico tiene consecuencias de

7 De este corpus global ya hay dos estudios parciales: Fernández Juncal y Hernández Muñoz (2019) sobre antropónimos y Fernández Juncal (en prensa) sobre nombres de marcas. 
tipo sintáctico y morfológico, como que apenas presenta variación interna (en todo caso, lexicalizada) y tampoco moción de género y número (Bajo Pérez 2002).

Las decisiones más importantes acerca de la edición de materiales son las siguientes:

a. Se respeta el valor denominativo del nombre del comercio, corrigiendo los posibles errores de denominación que el informante pudiera haber producido (aunque estos son mínimos y afectan a unidades menores de la denominación, como preposiciones o artículos), como Árbol en lugar de El Árbol, Jamonería El Carmen por La jamonería de Carmen o The Phone House por Phone House. Esto se debe al respeto por el nombre, registrado o no, pero que forma parte de la identidad del comercio (Alserhan y Alserhan 2012). En la enumeración de los informantes, sobre todo para los establecimientos de hostelería (bares y restaurantes), ha sido sobresaliente el empleo del artículo como rastro del componente omitido (El Niebla, El Gambrinus, El Gatsby), elemento que ha sido eliminado en la edición final.

b. Se ha empleado el paréntesis para aquellas lexías complejas, uno de cuyos integrantes es de carácter potestativo, dato que tiene importancia en la interpretación de los datos de las encuestas, como veremos: Audi + Concesionario Audi > (Concesionario) Audi; Avenida + Perfumerías Avenida $>$ (Perfumerías) Avenida, Levis + Levis Center $>$ Levis (Center).

c. De acuerdo con la norma ortográfica académica, todos los elementos han sido escritos con letra mayúscula.

d. Se conservan los nombres de marcas que no representan por sí mismas un establecimiento (Hacendado, Hunter, Menamano) y que, en cualquier caso, resultan minoritarias, apenas los tres casos mencionados. Asimismo, se conserva alguna creación ad hoc del informante: Los Chinitos, Calero.

Una vez editados los datos, han sido tratados con las herramientas que nos proporciona la plataforma dispolex.com y también con el programa estadístico SPSS (23). 


\section{ANÁLISIS DE RESULTADOS}

\subsection{El CENTRO DE INTERÉS NOMBRES DE COMERCIOS}

En la tabla 2 se encuentran los datos cuantitativos correspondientes a este nuevo centro de interés y lo contrastamos con los correspondientes al centro de interés Marcas, que tratamos en Fernández Juncal (en prensa).

\begin{tabular}{|lccccc|}
\hline & $\begin{array}{c}\text { Palabras } \\
\text { totales }\end{array}$ & $\begin{array}{c}\text { Vocablos } \\
\text { diferentes }\end{array}$ & $\begin{array}{c}\text { Vocablos } \\
\text { diferentes }>\mathbf{1} \\
\text { informante }\end{array}$ & $\begin{array}{c}\text { Palabras por } \\
\text { informante }\end{array}$ & $\begin{array}{c}\text { Índice de } \\
\text { cohesión }\end{array}$ \\
\hline Comercios & 995 & 472 & 123 & 16,58 & 0.04 \\
\hline Marcas & 1104 & 424 & 168 & 18.40 & 0.04 \\
\hline
\end{tabular}

Tabla 2. Índices cuantitativos totales

Si consideramos todos los índices cuantitativos, estamos ante centros con poca producción respecto a los campos nocionales tradicionales de los estudios de disponibilidad léxica. Las palabras totales para los comercios muestran una cifra inferior a la de marcas, pero hay más vocablos diferentes, aunque la mayor parte de estos son proporcionados por un solo informante (349 de comercios frente a 256 de marcas). A pesar de que ambos centros comparten el mismo índice de cohesión ${ }^{8}$, los datos citados nos permiten deducir mayor dispersión en el actual. Podemos adjudicar esta situación a que se trata de un campo nocional al que afectan de manera más notoria la experiencia y modo de vida personales, mientras que el otro campo está más influido por la presión global de la mercadotecnia, por lo que hay más puntos de encuentro en las respuestas de los informantes, aunque ambos en su conjunto poseen menos densidad que los centros que se emplean en el Proyecto Panhispánico. En consonancia con estos primeros datos, el promedio de respuestas, valor más significativo en la interpretación de las diferencias, es considerablemente inferior, lo que no ha de sorprender habida cuenta de que el primer centro podría considerarse en cierto modo un subtipo del segundo, donde abundan más unidades.

\footnotetext{
8 Recordemos que este valor se halla dividiendo el promedio de palabras por informante entre el número de vocablos o palabras diferentes, y marca, en un intervalo de 0 a 1 , el carácter disperso o compacto del campo cognitivo, es decir, el grado de coincidencia que muestran los informantes en un determinado centro de interés. Este valor nos permite ver la amplitud de cada uno de los centros, abiertos o difusos, cuando las respuestas son diferentes, o cerrados o compactos, cuando las respuestas son convergentes.
} 
Estamos antes dos campos léxicos muy entrelazados: por una parte, aunque de manera limitada, encontramos elementos idénticos compartidos: un $26,8 \%$ de los términos de ambos corpus son comunes. Hay también elementos cruzados (nombres de comercios en las listas de marcas, nombres de marcas en las listas de comercios). Esto se debe a que los nombres de comercios son también marcas; así los nombres de comercio implican el concepto de marca distintiva, existen marcas que poseen puntos de venta y los propios establecimientos son mayoritariamente distribuidores que alojan marcas en su seno.

La proyección de las variables independientes en el conjunto de resultados se detalla en la tabla 3 :

\begin{tabular}{|c|c|c|c|c|c|}
\hline & & $\begin{array}{c}\text { Palabras } \\
\text { totales }\end{array}$ & $\begin{array}{l}\text { Palabras } \\
\text { diferentes }\end{array}$ & $\begin{array}{c}\text { Palabras por } \\
\text { informante }\end{array}$ & $\begin{array}{l}\text { Índice de } \\
\text { cohesión }\end{array}$ \\
\hline \multicolumn{6}{|c|}{ Género } \\
\hline & hombres & 493 & 314 & 16,43 & 0,05 \\
\hline & mujeres & 502 & 235 & 16,73 & 0,07 \\
\hline \multicolumn{6}{|c|}{ Estudios } \\
\hline & Universitarios & 550 & 340 & 18,33 & 0,05 \\
\hline & $\begin{array}{l}\text { No } \\
\text { universitarios }\end{array}$ & 445 & 210 & 14,83 & 0,07 \\
\hline \multirow[t]{3}{*}{ Edad } & Generación 1 & 364 & 197 & 18,20 & 0,09 \\
\hline & Generación 2 & 333 & 209 & 16,65 & 0,08 \\
\hline & Generación 3 & 298 & 190 & 14,90 & 0,08 \\
\hline
\end{tabular}

Tabla 3. Datos desglosados de cada variable

Las cifras que encontramos para cada estrato son significativas, pero, si nos detenemos en los promedios de palabras por informante, podemos concluir que apenas hay diferencias en la producción de hombres y mujeres, pero sí hay disparidades significativas entre universitarios y no universitarios a favor de los primeros. Respecto a la edad, se produce una gradación, de forma que son los jóvenes los que aportan más respuestas de media mientras que la generación con edad más avanzada presenta un promedio muy inferior. Por su parte, la generación intermedia también muestra un índice intermedio.

Contrastamos estos datos con las pruebas estadísticas que hemos realizado empleando el programa SPSS (23). Como paso previo, hemos llevado a cabo las pruebas de normalidad estudiando la asimetría, la curtosis, los gráficos Q-Q y la prueba de normalidad Shapiro-Wilk (S-W), de las que se concluye que podemos asumir una distribución normal, por lo que aplicamos pruebas de tipo paramétrico a nuestro corpus. Así, la prueba T-Student para variables dicotómicas aporta un valor $\mathrm{p}=0,005(<0,05)$, que nos permite rechazar la hipótesis nula y confirmar la pertinencia de la variable estudios, de manera 
que hay diferencias en el comportamiento de ambos colectivos. Asimismo, ratificamos las similitudes entre hombres y mujer con la misma prueba, que ofrece un valor $\mathrm{p}=0,981(>0,05)$, que acepta la hipótesis nula. Sin embargo, la prueba de Anova de un factor que hemos aplicado para la variable edad, también confirma la hipótesis nula con un valor $\mathrm{p}=0,131(>0,05)$ a pesar de que los datos parecen indicar algún tipo de correlación.

En la tabla 4 se observan los índices de compatibilidad de conjuntos de acuerdo con esas mismas variables.

\begin{tabular}{|c|c|c|c|c|c|}
\hline & & & $\begin{array}{c}100 \\
\text { primeras } \\
\text { palabras }\end{array}$ & 0,02 de DL & $\begin{array}{l}80 \% \text { frecuencia } \\
\text { acumulada }\end{array}$ \\
\hline Género & & Comercios & 21,21 & 19,80 & 14,01 \\
\hline Estudios & & Comercios & 19,76 & 20,11 & 13,96 \\
\hline Edad & G1-G2 & Comercios & 22,70 & 18,02 & 16,59 \\
\hline & G1-G3 & Comercios & 17,65 & 15,98 & 13,27 \\
\hline & G2-G3 & Comercios & 18,34 & 16,16 & 12,81 \\
\hline
\end{tabular}

Tabla 4. Grado de compatibilidad de las listas de LD de acuerdo con las tres variables

Se confirma con estos valores, muy bajos en todos los casos, la percepción de que estamos ante un centro de interés de gran dispersión, más incluso que el de las marcas (Fernández Juncal en prensa). Puede servirnos como dato relativo de comparación que el índice de compatibilidad mínimo encontrado en Cantabria (Fernández Juncal 2013) para los centros de interés tradicionales en la variable género es del 44,6 (y llega hasta el 81,25 ) y el equivalente en la variable nivel sociocultural es del 30,49 (y alcanza el 89,7).

\subsection{LOS NOMBRES DE COMERCIOS DISPONIBLES}

A continuación, analizaremos los rasgos que caracterizan este tipo de nombres a partir del corpus de nombres de comercios disponibles eliminando aquellos que solo han sido mencionados por una sola persona. Se trata de 123 vocablos, apenas un tercio del total del conjunto de términos diferentes captados. Emplearemos dos puntos de vista para el análisis: el tipo de comercio y la forma lingüística. Antes de presentar los resultados conviene hacer dos precisiones: 
a) en muchas ocasiones los informantes añaden el tipo de comercio (antepuesto o pospuesto). Esto ocurre en dos circunstancias que tienen relación con el uso toponímico de este tipo de crematónimos:

a. cuando el nombre no posee rasgos individualizadores suficientes como para resultar identificable: Pescadería Paco, Frutería Ángel, Centro Comercial el Tormes.

b. Cuando el nombre de comercio coincide con una marca y se quiere señalar la diferencia entre ambos: Concesionario Toyota respecto a Toyota y Levis Center respecto a Levis.

b. Aparecen términos obsoletos que no se corresponden con comercios actuales, especialmente en los informantes de edad más avanzada (Simago, Pryca, Hijos de Primitivo Muñoz, Mantequerías Paco). Esto indica la vigencia de este tipo de términos en el lexicón de los informantes.

En la tabla 5 se desglosan los rasgos atendiendo al ámbito y procedencia de la empresa propietaria del comercio y la categoría de productos y servicios que ofrecen. Para este último criterio hemos seguido la tipología que establece García Marcos (2019) apoyándose en la Clasificación Internacional de Productos y Servicios de Niza (2012):

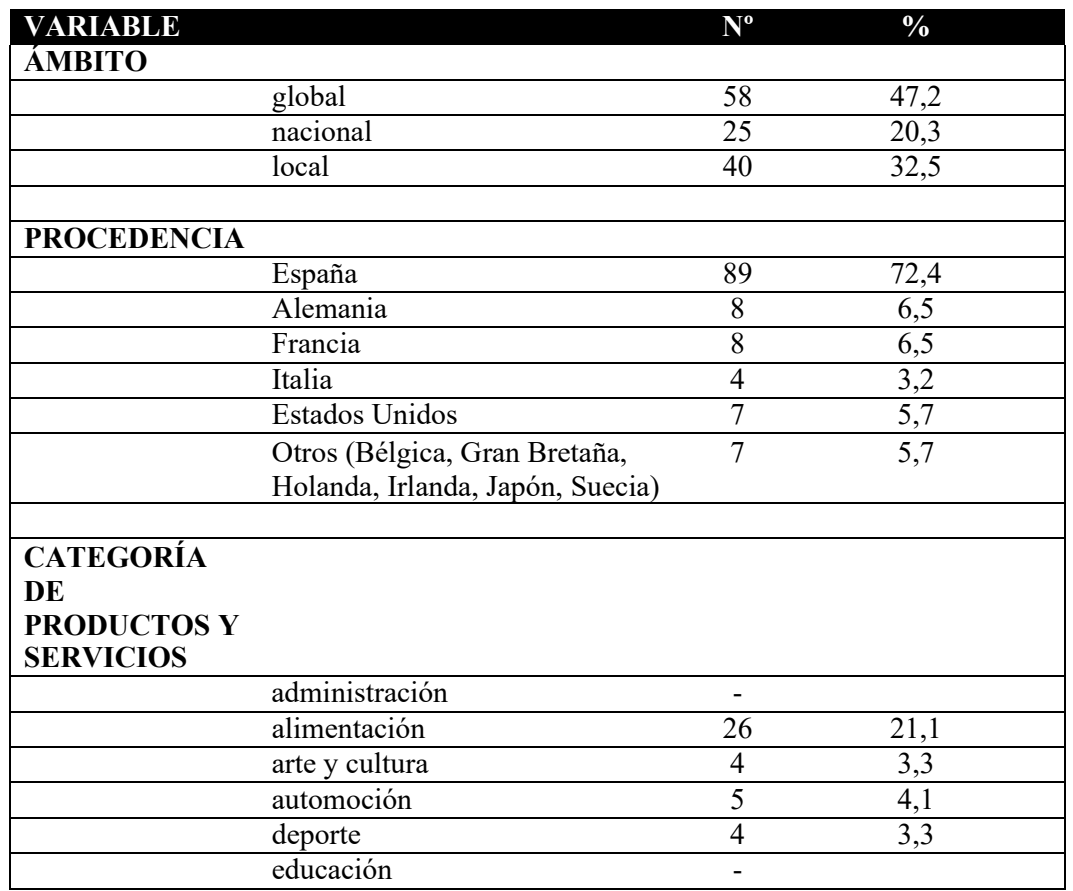




\begin{tabular}{|lcc|}
\hline estética & 5 & 4,1 \\
\hline gestión/finanzas & - & \\
\hline personal & - & \\
\hline hogar & 8 & 6,5 \\
\hline hostelería & 18 & 14,6 \\
\hline informática & 1 & 0,8 \\
\hline juguetería & 2 & 1,6 \\
\hline mantenimiento & - & \\
\hline multiproductos & 9 & 7,3 \\
\hline ocio y recreo & - & \\
\hline papelería & - & \\
\hline religión/espíritu & - & 30,9 \\
\hline ropa/complementos & 38 & \\
\hline sanidad & - & 2,4 \\
\hline sexo & - & \\
\hline telefonía & 3 \\
\hline transportes & - \\
\hline
\end{tabular}

Tabla 5. Clasificación del corpus por criterios de tipo empresarial

En primer lugar, la mayoría de los comercios son sede de empresas de origen español (casi dos tercios del total), pero la mayoría, casi la mitad, son de carácter internacional, asunto que tendrá su refrendo en los usos lingüísticos, que veremos más adelante. La homogeneización del paisaje urbano es asunto que ha sido percibido desde hace tiempo y que ahora es conocido como placelessness, concepto creado por Relph (1976) y desarrollado después en otros estudios de geografía humana y psicología social. Se trata de un fenómeno que ha sido puesto en cuestión y que ya ha suscitado en muchas grandes ciudades medidas de carácter político para prevenirlo y a favor de la conservación de la identidad que proporcionan los comercios no adscritos a marcas globales. No obstante, expertos como Sjöblom (2013) afirman que lo global y lo local no son conceptos en las antípodas sino perspectivas diferentes desde las que interpretar los acontecimientos sociales, dos lados de la misma moneda que se funden en la idea de lo glocal. En este sentido, Bergien (2012) nos recuerda que las grandes compañías intentan compensar la falta de rasgos identitarios (y estos sí los posee el comercio local) en favor del valor de la singularidad, evitando así la sensación de réplica que un producto podría adquirir en ausencia de rasgos diferenciadores de tipo cultural.

En nuestro corpus apenas un tercio de los establecimientos son de alcance local, lo que nos da una idea de los cambios que se han producido en la economía del intercambio de bienes y servicios. Bien es cierto que estas cifras son muy inferiores a las detectadas respecto a las marcas globales, que llegaban a un $86,9 \%$ del total del corpus. 
Si consideramos el país de procedencia, es España el que está ligado a más crematónimos, seguido a mucha distancia por países del mundo desarrollado, especialmente los más pujantes en el área europea. Las categorías de bienes y servicios que ofrecen los establecimientos no son tan variadas como las que se recogen en los trabajos de paisaje lingüístico. Así, como ocurría con las marcas, no hay mención de empresas dedicadas a los servicios (finanzas, sanidad, transportes), pero sí de bienes tangibles. Ropa y complementos, alimentación y hostelería son las esferas comerciales más citadas, seguidas más de lejos por productos del hogar, la estética, la cultura o la automoción. En este caso sí hay una diferencia con los resultados sobre marcas, ya que automoción desciende varios puestos desde el primero y son los lugares donde adquirir los bienes que afectan más a nuestra vida cotidiana los que parecen reseñados en las primeras posiciones.

En la tabla 6 se especifican las lenguas de origen de los crematónimos aportados y el fundamento de su denominación. La clasificación de los criterios denominativos es la empleada en Fernández Juncal (en prensa), basada en Danesi (2013), Jordá et al. (2010) y González del Río et al. (2011), así como en Pinillos et al. (2016):

\begin{tabular}{|c|c|c|c|}
\hline \multicolumn{4}{|l|}{ LENGUA } \\
\hline & Español & 63 & 51,2 \\
\hline & Inglés & 18 & 14,6 \\
\hline & Francés & 6 & 4,9 \\
\hline & $\begin{array}{l}\text { Italiano } \\
\end{array}$ & 5 & 4,1 \\
\hline & Alemán & 5 & 4,1 \\
\hline & Latín & 3 & 2,4 \\
\hline & $\begin{array}{l}\text { Otros (griego, hebreo, holandés, japonés, } \\
\text { sueco, vasco) }\end{array}$ & 8 & 6,5 \\
\hline & Creaciones & 6 & 4,9 \\
\hline & Desconocida & 5 & 4,1 \\
\hline & Mixto & 4 & 3,2 \\
\hline \multicolumn{4}{|l|}{$\begin{array}{l}\text { CRITERIO } \\
\text { DENOMINATIVO }\end{array}$} \\
\hline & Antropónimos & 36 & 30 \\
\hline & Asociativos & 33 & 26,8 \\
\hline & Descriptivos & 25 & 20,3 \\
\hline & Siglas y acrónimos & 12 & 9,8 \\
\hline & Artificiales & 6 & 4,9 \\
\hline & Topónimos y gentilicios & 5 & 4,1 \\
\hline & Desconocido & 5 & 4,1 \\
\hline & TOTAL & 123 & \\
\hline
\end{tabular}

Tabla 6. Clasificación del corpus por criterios lingüísticos 
El español es la lengua más empleada, pero casi la mitad de los rótulos de establecimientos está escrita en otras lenguas: el inglés de manera muy destacada y luego, de manera minoritaria, por otras, generalmente europeas 9 . Sí merece la pena destacar que no existe una concordancia entre estos datos y los correspondientes al país de origen de la empresa vendedora, de lo que se deduce que muchas firmas españolas emplean términos procedentes de otras lenguas para singularizar su comercio. El empleo del inglés en el ámbito global ha sido estudiado profusamente: Pennycook (2003) afirma que el inglés cumple un cometido de unificación en detrimento de las especificidades lingüísticas locales, transmitiendo además valores y estilos de vida que en muchas ocasiones resultan ajenos a la comunidad donde se implantan. Por su parte, Friedrich (2002) compendia las ventajas que ofrece el uso del inglés respecto a otras lenguas en las siguientes: simboliza modernidad y aporta connotaciones de occidentalización, es más inteligible que otras lenguas, posee características intrínsecas (como la longitud de sus palabras) que se ajustan a los requisitos de la comunicación comercial $\mathrm{y}$ publicitaria $\mathrm{y}$, además, ofrece un nuevo repertorio para las necesidades creativas de los publicistas. Wong y Leben (2012) afirman que, de hecho, de acuerdo con los últimos estudios, los vocablos de origen inglés son preferidos por los consumidores por encima de palabras creadas ex nihilo aunque también presentan problemas: el dominio del inglés es escaso por parte de muchos consumidores, en algunas comunidades se detecta cierta resistencia a su uso y además algunos términos en esa lengua pueden ofrecer interferencias semánticas y connotativas respecto a la lengua nativa del lugar.

En cualquier caso, los resultados del corpus confirman el carácter multilingüe y multicultural del paisaje lingüístico de nuestras ciudades. Las nuevas condiciones del comercio internacional implican la creación de redes más extensas e internacionales, con una gran movilización de productos y servicios, apoyada en una base tecnológica avanzada y con implicaciones de carácter cultural que traspasan fronteras (Chiang 2009: 330). En ese medio las empresas buscan nombres con connotaciones positivas de actualidad, internacionalidad e innovación. Boerrigter y Nijboer (2012), citando a Nunberg, recuerdan el impacto que han tenido los intensos procesos de globalización en la denominación de los lugares comerciales, hasta el punto de hablar de una nueva lengua internacional, una lingua branda, que permite

9 Baños Maniega (2018) encuentra en su estudio de la onomástica comercial de tres zonas de Salamanca y cuatro sectores comerciales una distribución diferente, de forma que los rótulos en español alcanzan el 73,7\% del total. 
la comunicación internacional y que simultáneamente debe acomodarse al lugar donde se instala.

Si tenemos en consideración los procedimientos de creación del crematónimo, el recurso más habitual es el empleo de antropónimos, generalmente del fundador del negocio, bien sea su nombre de pila, su hipocorístico, el apellido o el nombre completo (Frutería Ángel, Carnicería Juanma, Lidl, Yves Rocher), más frecuentes los dos primeros en el ámbito local. Se trata de un mecanismo cuya extensión ha sido puesta de manifiesto en diversos estudios y, de acuerdo con Pinillos et al. (2016), muy productivo en el ámbito español, aunque, según algunas investigaciones (Leibring 2012), su uso ha ido decayendo con el tiempo. En otras ocasiones el antropónimo es ficticio o alterado (Massimo Dutti, Roberto Verino). Ambas circunstancias han sido interpretadas como una forma de antropomorfización de las señales con el objeto de humanizar y acercar el establecimiento a los posibles usuarios (Hakala et al. 2015). En la tabla 7 clasificamos los 36 antropónimos encontrados:

\begin{tabular}{|lccc|}
\hline \multicolumn{1}{|c}{ Estructura antropónimos } & TOTAL & DE HOMBRE & DE MUJER \\
\hline Nombre & 8 & 4 & 4 \\
\hline Nombre + apellido & 9 & 8 & 1 \\
\hline Apellido(s) & 12 & 12 & 1 \\
\hline Hipocorístico & 3 & 3 & - \\
\hline Acrónimo o siglas & 4 & 4 & - \\
\hline
\end{tabular}

Tabla 7. Estructura y aplicación de género de los antropónimos del corpus

Como comprobamos, la presencia de nombres de mujer es escasa y especialmente poco significativa cuando se trata de un apellido. Estos datos confirman la tendencia que ya observamos en Fernández Juncal (2002 y 2018) y los hallados por Leibring (2012: 55):

Another aspect that has to be taken into account is the long-established custom of referring to women, even well-known women, with their first names, and to men with their surnames (Milles 2008:19), a habit which may influence the name giving of companies.

Son también fórmulas habituales el empleo de términos con asociación positiva hacia los bienes en venta (Women's Secret, La Montanera), seguido por aquellos que los describen (Telepizza, Prenatal, Taberna Celta, El Burger). Se recurre también a los acrónimos y siglas (Fremar, Gadis, Covirán) y, ya en menor medida, a los topónimos (Perfumerías Avenida, El Tormes, Carrefour), y las creaciones ad hoc (Bershka, Oysho). Los topónimos, tanto mayores como menores, suelen emplearse para aportar una connotación del origen de la compañía, el vínculo de esta con un cierto 
lugar, con una intención sobre el comportamiento del consumidor menos informativa que emocional (Bergien 2012). El origen locativo de algunos nombres de comercios refuerza su función complementaria como topónimos, pero no es condición indispensable para que desempeñen ese papel.

Los resultados correspondientes al criterio de denominación son paralelos a los obtenidos en las marcas; así, el peso de los distintos recursos empleados es muy similar y sigue prácticamente el mismo orden de prelación.

Sjöblom (2005) divide los nombres de compañías en tres tipos: informativos, asociativos y sin sentido (meaningless). Leibring (2012) sostiene que tradicionalmente se ha empleado más el primero, pero que las tendencias están cambiando hacia el segundo y el tercero, que se están instalando en los establecimientos más recientes. En nuestro corpus predomina todavía la función informativa, pero se percibe la aparición de creaciones y nombres de carácter asociativo (Camelot, Gatsby), sobre todo en empresas con expansión internacional (Zara, Intimissimi). De hecho, si sumamos los casos de creaciones con los términos cuyo origen ha sido imposible establecer y que, en cualquier caso, resultan opacos (Pimkie, Nyx), llegaríamos a un $9 \%$ del total.

Por último, en lo que se refiere a la extensión de los crematónimos, la mayoría $(62,6 \%)$ están formados por un solo elemento, proveniente de un nombre propio previo (Pascual, Lidl), de un nombre común (Peatón, Casa), de siglas o acrónimos (Seat, Udaco) y ya de forma más ocasional, de adjetivos (Intimissimi) e incluso de onomatopeyas (Boom). El 37,4\% de las entradas de nuestro corpus está constituido por una lexía compleja, mayoritariamente un sintagma nominal (La Tahona de la Abuela, El Árbol, Women's secret) pero también de manera esporádica una frase (Toys " $R$ " us, Pull\&Bear).

Para completar este apartado aportamos en la tabla 8 los 25 nombres de comercios más disponibles de nuestro corpus,

\begin{tabular}{|llccc|}
\hline & Disponibilidad & $\begin{array}{c}\text { Frecuencia } \\
\text { relativa }\end{array}$ & \% aparición \\
\hline $\mathbf{1}$ & Zara & 0.40626 & $3.827 \%$ & $63.333 \%$ \\
\hline $\mathbf{2}$ & Carrefour & 0.40458 & $3.323 \%$ & $55.000 \%$ \\
\hline $\mathbf{3}$ & Mercadona & 0.34535 & $3.021 \%$ & $50.000 \%$ \\
\hline $\mathbf{4}$ & DIA & 0.30335 & $2.618 \%$ & $43.333 \%$ \\
\hline $\mathbf{5}$ & El Corte Inglés & 0.26343 & $2.316 \%$ & $38.333 \%$ \\
\hline $\mathbf{6}$ & Mango & 0.19120 & $1.813 \%$ & $30.000 \%$ \\
\hline $\mathbf{7}$ & Bershka & 0.17803 & $1.611 \%$ & $26.667 \%$ \\
\hline $\mathbf{8}$ & Stradivarius & 0.16167 & $1.611 \%$ & $26.667 \%$ \\
\hline $\mathbf{9}$ & E. Leclerc & 0.15544 & $1.511 \%$ & $25.000 \%$ \\
\hline $\mathbf{1 0}$ & H\&M & 0.15416 & $1.611 \%$ & $26.667 \%$ \\
\hline $\mathbf{1 1}$ & El Arrbol & 0.15331 & $1.410 \%$ & $23.333 \%$ \\
\hline $\mathbf{1 2}$ & Gadis & 0.14315 & $1.410 \%$ & $23.333 \%$ \\
\hline
\end{tabular}




\begin{tabular}{|lllll|}
\hline $\mathbf{1 2}$ & Gadis & 0.14315 & $1.410 \%$ & $23.333 \%$ \\
\hline $\mathbf{1 3}$ & Media Markt & 0.14024 & $1.611 \%$ & $26.667 \%$ \\
\hline $\mathbf{1 4}$ & (Congelados) La Isla & 0.12529 & $1.007 \%$ & $16.667 \%$ \\
\hline $\mathbf{1 5}$ & Decathlon & 0.12098 & $1.208 \%$ & $20.000 \%$ \\
\hline $\mathbf{1 6}$ & Cortefiel & 0.10512 & $1.108 \%$ & $18.333 \%$ \\
\hline $\mathbf{1 7}$ & Pull\&Bear & 0.10472 & $1.108 \%$ & $18.333 \%$ \\
\hline $\mathbf{1 8}$ & Leroy Merlín & 0.10298 & $1.309 \%$ & $21.667 \%$ \\
\hline $\mathbf{1 9}$ & Lidl & 0.10219 & $1.007 \%$ & $16.667 \%$ \\
\hline $\mathbf{2 0}$ & Covirán & 0.09113 & $0.806 \%$ & $13.333 \%$ \\
$\mathbf{2 1}$ & (Perfumerías) & 0.08921 & $1.007 \%$ & $16.667 \%$ \\
& Avenida & & \\
\hline $\mathbf{2 2}$ & Aldi & 0.08464 & $0.705 \%$ & $11.667 \%$ \\
\hline $\mathbf{2 3}$ & Décimas & 0.06059 & $0.705 \%$ & $11.667 \%$ \\
\hline $\mathbf{2 4}$ & Massimo Dutti & 0.06039 & $0.504 \%$ & $8.333 \%$ \\
\hline $\mathbf{2 5}$ & (Galerías) José Mari & 0.06008 & $0.604 \%$ & $10.000 \%$ \\
\hline
\end{tabular}

Tabla 8. 25 nombres de comercios más disponibles

\section{CONCLUSIONES}

Los nombres de comercios presentan las dificultades de análisis de los nombres de marcas, con los que, por otra parte, tienen establecidos paralelismos indudables: ambos pertenecen a lo que en onomástica se denominan crematónimos o ergónimos. En segundo lugar, desde el punto de la disponibilidad léxica, estamos ante campos nocionales con comportamientos semejantes: con producción media baja (inferior aún para los comercios), difusos, sobre todo si examinamos el grado de compatibilidad de conjuntos, con niveles de coincidencia entre estratos muy inferiores a los detectados en estudios previos. Sin embargo, si analizamos la influencia de las variables seleccionadas, los nombres de comercio están afectados por la variable estudios, pero, al contrario que el otro centro, no parece que el género o la edad sean decisivos para explicar las diferencias cuantitativas entre los informantes. Respecto a la edad, sí se observa una cierta gradación que hace que los más jóvenes ofrezcan resultados más elevados mientras que los mayores presentan menos aportaciones.

Desde el punto de vista cualitativo, también hay puntos en común: más de un cuarto de los elementos $(26,8 \%)$ de los nombres de comercios son compartidos con los del corpus de marcas. Este grado de coincidencia aumenta si además contabilizamos otros nombres de marca que no están en el corpus de marcas disponibles, de forma que llegaríamos a la cifra de 49,6\% 
de marcas dentro de los nombres de comercios. Por lo tanto, hay una gran mayoría de marcas que cuentan con establecimientos propios o en régimen de franquicias a los que han hecho extensivo el nombre que les sirve como identificador mientras que muy poco más de la mitad de los casos pueden considerarse comercios distribuidores como tal.

Este solapamiento entre ambas categorías se prolonga al examinar otros rasgos: estamos ante dos tipos de vocablos que comparten el carácter multilingüe, con la incorporación de elementos de otras lenguas, que aportan valores connotativos y simbólicos, especialmente en el caso del inglés, que añade atractivos como la internacionalidad o la innovación.

Asimismo, coinciden ambas clases en los criterios motores en la creación de las nuevas denominaciones: los antropónimos son una inagotable fuente de formación de marcas y nombres de comercios. Su empleo viene de antiguo, transmiten ideas de tradición y solvencia y, sobre todo, humanizan los productos. Sin embargo, parece que su empleo en otras sintopías está en declive y como se deduce también de nuestro catálogo, refleja en su selección de nombres una sociedad de carácter patriarcal, donde los antropónimos de mujer desempeñan un papel muy limitado y asimétrico.

También otros criterios son la base de formación de nombres de marcas y comercios: los nombres que son elegidos por las sugerencias que proporcionan conviven con los nombres que ayudan a describir los productos o servicios que se pretenden anunciar. También intervienen otros recursos: los neologismos, las siglas o acrónimos, que han perdido en casi todos los casos su referencia original, y los topónimos, que favorecen el anclaje emocional e histórico de la empresa en cuestión.

Marcas y comercios también presentan discrepancias en nuestro corpus de disponibilidad de acuerdo con su carácter como entidades físicas o abstractas. Las denominaciones de marcas disponibles poseen mucha más extensión geográfica y abundan aquellas que son de carácter internacional. Son también transnacionales las que más proliferan en el caso de los comercios como consecuencia inevitable de la globalización en el desarrollo de la venta e intercambio de bienes y servicios, pero, como era de esperar, hay considerable mayor presencia de comercios locales $\mathrm{y}$, ocupando un tercer lugar, los de ámbito regional o nacional. Esto hace que el porcentaje de establecimientos españoles doble al de marcas españolas y presente menor variedad de países de procedencia involucrados, ya que los comercios multimarcas absorben la distribución de los distintos productos.

La importancia concedida a los establecimientos que comercian con ropas y complementos, alimentación y los establecimientos de hostelería se explica por la presencia de estos en la vida cotidiana frente a las marcas 
de automoción, que destacaban entre las marcas en conexión directa con el volumen de recursos destinados a su publicidad.

Los nombres de comercios establecen una conexión inevitable con su entorno, no solo con el paisaje lingüístico sino con el entorno urbano público donde se desarrolla su función, que va más allá de la identificación de una empresa. En muchas ocasiones sustituye la función de los topónimos menores y se convierten en referencias de localización, hasta alcanzar en ocasiones estatus emblemático. Esta adquisición de competencias toponímicas es fenómeno observado en muy diferentes sintopías, no solo a través de nombres propios, como es el caso, sino por otros métodos (posiciones geográficas, referencias respecto a personas o referencias a otro lugar (Ainiala 2014). Así, en la ciudad de Salamanca funcionan de esta manera nombres como $E l$ reloj (del Ayuntamiento en la Plaza Mayor), El Toscano (cafetería situada en una intersección) o El Corte Inglés (para designar una zona). Esta naturaleza multifuncional hace que la clasificación de los nombres de comercio haya sido confusa, más desde su índole toponímica en su categoría de nombre propio, más como crematónimo como objeto de estudio socionomástico. Desde esta última perspectiva se puede apreciar la confluencia de elementos descriptivos de la realidad social: lo local frente a lo global (o, lo que es lo mismo, lo identitario frente a lo general), el multilingüismo, la diversidad funcional y los cambios que se producen en los gustos y valores del conjunto social.

\section{REFERENCIAS BIBLIOGRÁFICAS}

Ainiala, Tehri. 2014. Identifying places and Discussing names: the use of toponyms in a Conversation. En Betina Löfström y Jonas Schnabel-Le Corre (eds.). Challenges in Synchronic Toponymy, pp. 33-46. Tübingen: Narr Francke Attempto Verlag.

Alserhan, Baker Ahmad y Zeid Ahmad Alserhan. 2012. Naming businesses: names as drivers of brand value. Competitiveness Review: An International Business Journal 22 (4): 329-342.

Amorós Negre, Carla, Carmen Fernández Juncal, Natividad Hernández Muñoz y Emilio Prieto de Los Mozos. 2012. Difficulties in defining the standard Spanish lexicon. En Rudolf Muhr (ed.). Non-Dominant Varieties of Pluricentric Languages. Getting the Picture. In memory of Prof. Michael Clyne, pp. 61-80. Viena: Peter Lang Verlag.

BAjo PÉRez, Elena. 2002. La caracterización morfosintáctica del nombre propio. Noia: Editorial Toxosoutos.

Baños Maniega, Elisa. 2018. Onomástica comercial. Estudio del paisaje lingüístico de la ciudad de Salamanca. Trabajo de fin de grado, Universidad de Salamanca.

Bauer, Gerhard. 1985. Namenkunde des Deutschen. Bern/Frankfurt: Peter Lang. 
Bergien, Angela. 2012. Cultural and Regional Connotations of Company Names in Local Contexts. En R. Boerrigter y H. Nijboer (eds.), pp. 7-12.

Boerrigter, Reina y Harm Nijboer (eds.). 2012. Names as Language and Capital. Proceedings Names in the Economy III, Amsterdam, 11-13 June 2009. Disponible en http://www.meertens.knaw.nl/nite/ [Consulta 20 de febrero de 2019].

Boerrigter, Reina y Harm NiJboer. 2012. Preface: Names as language and capital. En Reina Boerrigter y Harm Nijboer (eds.), pp. 3-6.

CHIANG, SHIAO-YUN. 2009. Interformative meaning of signs: brand naming and globalization in China. Social Semiotics 19 (3): 329-344.

Cotticelli Kurrasa, PaOla, Alfredo TrovatoB y Vania Vigolo. 2012. Brand Name and Brand Image between Linguistics and Marketing. En R. Boerrigter y H. Nijboer (eds.), pp. 13-31.

DANESI, MARCEL. 2013. Semiotizing a product into a Brand. Social Semiotics 23 (4): 464-476.

Fernández Juncal, Carmen. 2002. Algunos datos socionomásticos de una comunidad de la región funcional de Salamanca. En J.A. Bartol et al. (eds.). Nuevas aportaciones al estudio de la lengua española. Investigaciones filológicas, pp. 257-264. Salamanca: Luso-Española de Ediciones.

2013. Léxico disponible de Cantabria. Salamanca: Universidad de Salamanca/ Universidad de Cantabria.

2018. Evolution of Anthroponyms in an Area of Linguistic Transition: a SocioOnomastic Study. Names: A Journal of Onomastics 66 (2): 85-95.

(en prensa). Los nombres de marca: disponibilidad léxica y caracterización. En RILCE. Revista del Instituto de Lengua y Cultura Española 37.1.

Fernández Juncal, Carmen y Natividad Hernández Muñoz. 2019. Disponibilidad léxica y socionomástica. OGIGIA 25: 185-210.

Fernández Leborans, María Jesús. 1999. El nombre propio. En Ignacio Bosque y Violeta Demonte (eds.). Gramática descriptiva de la lengua española. 1 Sintaxis básica de las clases de palabras, pp. 77-128. Madrid: Espasa Calpe.

Fèvre-Pernet, Christine. 2007. Onomastique commerciale et genre polysémiotique: les catalogues de jouets. Tesis doctoral, Université Toulouse le Mirail - Toulouse II.

2008. Stratégies dénominatives en onomastique commerciale. En Durand J. Habert B., Laks B. (eds.). Congrès Mondial de Linguistique Française - CMLF'08, pp. 15391550. Disponible en http://dx.doi.org/10.1051/cmlf08268 [Consulta 10 de abril de 2019].

Fèvre-Pernet, Christine y Michel Roché. 2005. Quel traitement lexicographique de l'onomastique commerciale? Pour une distinction Nom de marque/Nom de produit. Corela. Disponible en https://philpapers.org/rec/FVRQTL [Consulta 10 de mayo de 2019].

Frey Pereyra, M. L. H. 2007. Disponibilidad léxica y escritura del español como lengua extranjera: propuesta de comparación de dos corpus. Interlingüistica 17: 366-373.

Friedrich, Patricia. 2002. English in advertising and brand naming: sociolinguistic considerations and the case of Brazil. English Today 18 (3): 21-28.

GaLKowSKI, ARTUR. 2014. La nature idiosyncrasique des chrématonymes. En J. Tort i Donada y M. Montagut i Montagut (eds.). Els noms en la vida quotidiana. Actes del XXIV Congrés Internacional d'ICOS sobre Ciències Onomàstiques, pp. 150-58. Barcelona: Generalitat de Catalunya. Disponible en <http://www.gencat.cat/llengua/BTPL/ICOS2011 > [Consulta 10 de mayo de 2019].

García Marcos, Francisco. 2019. El lenguaje de las rotulaciones de establecimientos comerciales en las ciudades contemporáneas. Los casos de Almería, Lódź y Tarrasa. Signa 28: 699-732. 
González del Río, Jimena, Olga Ampuero Canellas, Begoña Jordá Albiñana y Teresa Magal Royo. 2011. El nombre de marca: interrelación de factores lingüísticos y corporativos. Revista de Lingüística y Lenguas Aplicadas 6: 181-93.

Gorter, Durk. 2014. Linguistic Landscape studies. En Jan-Ola Östman, Jef Verschueren (eds.). Handbook of pragmatics. 2014 Installment, pp. 1-34. Amsterdam: John Benjamins.

GRANDINETTI, R. 2008. Il rapporto tra produzione e consumo in una prospettiva storica. En R. Grandinetti (ed.). Marketing. Mercati, prodotti e relazioni, pp. 23-42. Roma: Carocci editore.

Gutknecht C. y E. Wehking. 1985. Produktnamen. Eine eigene Subklasse konkreter Substantive? I.T.L. Review of Applied Linguistics 67/68: 77-89.

Hakala, Ulla, Paula Suöblom y Satu-Paivi Kantola. 2015. Toponyms as carriers of heritage: implications for place branding. Journal of Product \& Brand 24: 263-275.

Hernández Muñoz, Natividad. 2010. El cambio de canal en la producción léxica experimental. LEA XXXII/2: 141-71.

Jordá Albiñana, Begoña, Olga Ampuero-Canellas, Jimena GonZÁlez-Del-Río y Teresa Magal-Royo. 2010. Análisis lingüístico de los nombres de marca españoles. Revista de Lingüística y Lenguas Aplicadas 5: 77-88.

Kremer, Ludger y Elke Ronneberger-Sibold (dirs.). 2007. Names in Commerce and Industry: Past and Present. Berlin: Logos.

KriPKe, SAul. 1981. Naming and Necessity. Oxford: Blackwell.

KryUKova, IRINA. 2012. Rebranding in Russian Ergonymy as a Matter of Sociolinguistics. En R. Boerrigter y H. Nijboer (eds.), pp. 41-47.

Leibring, Katharina. 2012. From Backmans Blommor to Hairstyle by Lena: The Use of Personal Names in the Names of Small Companies in Sweden. En R. Boerrigter y H. Nijboer (eds.), pp. 48-57.

Li, Fengru. 2012. A sociolinguistic inquiry into Chinese brand naming behaviors: moral dilemmas, constraining hieroglyphs and economic aspirations. En R. Boerrigter y H. Nijboer (eds.), pp. 58-63.

Líbano Zumalacárregui, M. A. 1993. Toponimia menor: onomástica comercial. Fontes Linguae Vasconum 62: 125-136.

Manjón-Cabeza Cruz, A. J. 2010. Aproximación a la organización semántica del léxico sobre juegos y diversiones. Estudios de Lingüística 24: 199-224.

Ministerio de Industria, Comercio y Turismo / Oficina Española de Patentes y Marcas. 2019. Cuestiones Básicas acerca de Marcas y Nombres Comerciales. Disponible en https:// bit.ly/2VBJf0s [Consulta 10 de mayo de 2019].

Moreu-Rey, E. 1974. Toponimia urbana i onomástica vária. Palma de Mallorca: Moll.

Moutinho, LuRdes de CASTRO y Rosa Lídia Coimbra. 1998. O Nome é a Alma do Negócio: Estudo Lingüístico dos Nomes das Lojas em Portugal. En Actas do XIII Encontro da Associação Portuguêsa de Linguística, II Vol., pp. 93-104. Lisboa: Colibri.

PenNyCoOK, Alastair. 2003. Global Englishes, Rip Slyme and performativity. Journal of Sociolinguistics 7 (4): 513-533.

Pinillos Laffón, Alberto, Fernando Olivares Delgado y Daniel Rodríguez Valero. 2016. El nombre de la marca corporativa. Una taxonomía de los nombres de empresa familiar en España. Revista Latina de Comunicación Social 71: 750-774.

Pottier, B. 1954. Aspects de l'onomastique: l'onomastique mineure. Revue Internationale d'onomastique 6 (1): 43-51.

PuZeY, G. 2011. New research directions in toponomastics and linguistic landscapes. Onoma 46: 211-226.

Real ACAdemia Española/ASALE. 2009. Nueva gramática de la lengua española. Madrid: Espasa. 
2010. Ortografía de la lengua española. Madrid: Espasa.

Relph, Edward. 1976. Place and Placelessness. London: Pion.

SCHIPPAN, THEA. 1989. Markennamen-Linguistische Probleme und Entwicklungstendenzen. Beiträge zur Erforschung der deutschen Sprache 9: 48-55.

SEIDE, M.S. y P. LuCAs. 2018. Os topônimos comerciais da cidade de Naranjal, Paraguai. Confluência 54: 164-195.

SJöBlom, Paula. 2005. The problem of meaning and function relating to company names. En Eva Brylla y Mats Wahlberg (eds.). Proceedings of the 21st International Congress of Onomastic Sciences. Uppsala 19-24 Aug. 2002. Vol. 1, pp. 264-276. Uppsala: Språkoch folkminnesinstitutet.

2013. Lumia by Nokia, iPhone by Apple: Global or Local Features in Commercial Names? En Paula Sjöblom, Terhi Ainiala y Ulla Hakala (eds.), pp. 2-14.

2016. Commercial names. En Carole Hough (ed.). The Oxford Handbook of Names and Naming, pp. 453-465. Oxford: Oxford University Press.

Suöblom, Paula, Terhi Ainiala y Ulla Hakala (eds.). 2013. Names in the Economy: Cultural Prospects. Newcastle upon Tyne: Cambridge Scholars.

2013. Preface. En Paula Sjöblom, Terhi Ainiala y Ulla Hakala (eds.), pp. viii-xii.

SMIRNOVA, NonNA. 2017. Naming in the public sphere of Stavropol city: sacred and profane. En Oliviu Felecan (ed.). Proceedings of ICONN 4, pp. 1048-1057. Cluj Napoca: Mega/ Argonaut.

Teutsch, Andreas. 2012. Speakability of Trademarks. En Boerrigter y Nijboer (eds.), pp. 82-92.

Van Langendonck, Will. 2007. Trade and Brand Names: Proper or Common Nouns? En Ludger Kremer y Elke Ronneberger-Sibold (dirs.), pp. 23-33.

Vande Casteele, Anne. 2012. A Semantic Description of Company Names in Spanish Business-related Newspaper Articles. En R. Boerrigter y H. Nijboer (eds.), pp. 93-103.

Weyers, Christian. 2007. Brandname, Herkunftsangabe und Freizeichen im Grenzbereich zwischen Proprium und Appellativum. En Ludger Kremer y Elke Ronneberger-Sibold (dirs.), pp. 45-60.

Wong, ANDREW y Will LeBen. 2012. English words in international brand names: proceed with caution. En R. Boerrigter y H. Nijboer (eds.), pp. 104-110. 